RESEARCH

\title{
Homocysteine is an indicator of arterial stiffness in Chinese women with polycystic ovary syndrome
}

\author{
Xia Wu ${ }^{1, *}$, Zhiling $\mathrm{Li}^{2}, *$, Wenjiang Sun ${ }^{3}$ and Huan Zheng ${ }^{4}$ \\ 'Department of Endocrinology, Jing'an District Centre Hospital of Shanghai (Huashan Hospital Fudan University Jing'an Branch), Shanghai, China \\ 2Department of Pharmacy, Shanghai Children's Hospital, Shanghai Jiao Tong University, Shanghai, China \\ ${ }^{3}$ Department of Rehabilitation, Shanghai General Hospital, Jiaotong University, Shanghai, China \\ ${ }^{4}$ Department of Cardiology, Worldpath Clinic International, Shanghai, China \\ Correspondence should be addressed to H Zheng: drzhenghuan@163.com \\ *(X Wu and Z Li contributed equally to this work)
}

\begin{abstract}
Polycystic ovary syndrome (PCOS) is associated with an increased risk of cardiovascular disease in women. Hyperhomocysteinemia ( $\mathrm{H}-\mathrm{Hcy}$ ) is closely related to arterial stiffness (AS) in patients with cardiovascular disease. This study aimed to investigate the relationship between serum homocysteine(Hcy) level and brachial-ankle pulse wave velocity (baPWV) in Chinese women with PCOS. A total of 124 PCOS women were enrolled and divided into two groups according to their baPWV values: normal, baPWV $<1400 \mathrm{~cm} / \mathrm{s}$ and high AS, baPWV $\geq 1400 \mathrm{~cm} / \mathrm{s}$. Univariate analysis was performed to investigate the relative factors for baPWV, and multiple regression analysis was used to evaluate the association of Hcy with baPWV. The group with high AS $(n=35)$ had higher Hcy levels than the other group $(n=89 ; P<0.05)$. Moreover, univariate analysis revealed that serum Hcy was positively correlated with baPWV $(r=0.133, P<0.01)$. In multiple regression analysis, the age-adjusted serum Hcy level was positively correlated with baPWV $(\beta=0.201$, $P<0.01)$. It remained positively associated with baPWV $(\beta=0.145, P<0.01)$ after further adjustments for age, BMI, PCOS duration, systolic blood pressure, and homeostasis model assessment-insulin resistance as well as several other factors correlated with baPWV. Our results demonstrated that $\mathrm{H}-\mathrm{Hcy}$ was significantly and independently related to elevated baPWV, suggesting that Hcy might play a role in the pathologic process of AS in women with PCOS. Further researches with more subjects are needed to explore whether Hcy would be a promising biomarker for the stratification management of PCOS women.
\end{abstract}

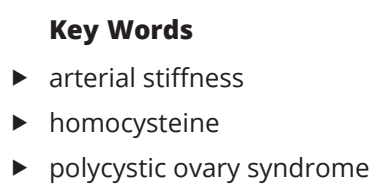

\section{Introduction}

Polycystic ovary syndrome (PCOS) is one of the most common endocrine disorders, affecting $6-21 \%$ of women of reproductive age depending on the population studied and the diagnostic criteria applied (1). It is characterized by hyperandrogenism and chronic anovulation. A diagnosis of PCOS has lifelong implications and is associated with an increased risk of type 2 diabetes, metabolic disorder, and cardiovascular disease(CVD) (2). The clinical symptoms of
PCOS include acne, amenorrhea or oligomenorrhea, and infertility, which tend to be the primary focus of clinical management. However, the impact of PCOS on future CVD risk should not be overlooked, and opportunities to implement CVD prevention strategies in these women should be prioritized (3). Arterial stiffness(AS) is known to play a predictive role in CVD (4), and clinical data show that AS is increased in women with $\operatorname{PCOS}(5,6,7)$.

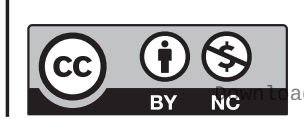


Automated computer-assisted brachial-ankle pulse wave velocity (baPWV) is a diagnostic method to investigate AS that has been recommended in large-scale sample studies (4). Over the past few decades, it has also been used in PCOS populations $(8,9)$.

Hyperhomocysteinemia (H-Hcy) is considered as an independent risk factor for the atherogenic and thrombotic components of arterial systems, although the definition of H-Hcy is not consistent in different publications, which used the cutoffs of more than 10,12 , or $15 \mu \mathrm{mol} / \mathrm{L}$ to describe H-Hcy $(10,11,12)$. However, substantial evidence shows that H-Hcy in PCOS women are associated with insulin resistance, excess androgens, abnormal heart rate recovery, and recurrent pregnancy loss (13). Furthermore, epidemiological studies have demonstrated that H-Hcy is significantly related to advanced AS in patients with hypertension (14), diabetes (15), and also in communitybased asymptomatic young adults (16). In China, similar investigations have supported the close correlation between H-Hcy and AS in community-based elderly people (17), hypertensive subjects (18), and women with a history of preeclampsia (19). In the past decades, a subtype of hypertension, H-type hypertension (hypertension with $\mathrm{H}-\mathrm{Hcy}$ ), has gained great attention in our country (12). It has been documented that $\mathrm{H}$-type hypertensive patients present with more macro/microvascular detriments compared with hypertensive patients having normal Hcy. Moreover, it was found that a combination of Hcy-lowering and anti-hypertension therapy significantly reduced the risk of stroke in the Chinese population, compared with anti-hypertension therapy alone (12). Based on those encouraging findings, we designed this study to determine whether H-Hcy would be a risk factor of AS in this specific female population, then to discuss the possibility of including Hcy level as a biomarker for the serial stratified prevention and management of CVD in those women.

\section{Subjects and methods}

\section{Subjects}

A total of 124 women who were diagnosed with PCOS according to the Rotterdam ESHRE-ASRM-sponsored PCOS criteria (20) at the Department of Gynecology and Obstetrics from January 2016 to December 2020 were enrolled in the study. Participants were excluded if they had any of the following: pregnancy, malignancy, liver or renal failure, acute systemic illness such as fever or acute infection, taking the supplement of folic acid regularly.
All procedures were approved by the Ethics Committee of Jing'an District Center Hospital of Shanghai Affiliated to Fudan University, and all participants gave their informed consents.

\section{Sample size}

The sample size was determined based on preliminary published clinical studies evaluating the relation between Hcy and AS or plasma markers of CVD in PCOS patients (13). The sample size was calculated with the formula: $N=\left(\left(Z_{\alpha}+Z_{\beta}\right) / C\right)^{2}+3$ described by Hulley et al. using the $\mathrm{r}$-value from Hcy and AS correlation tests (21). Assuming two-tailed $\alpha$ value $=0.05$ and $\beta=0.2$, minimum total sample size suggested was 92 PCOS patients.

\section{Clinical data collection}

Clinical data including medical history (hypertension is defined as systolic blood pressure (SBP) $\geq 140 \mathrm{mmHg}$ and/or diastolic blood pressure (DBP) $\geq 90 \mathrm{mmHg}$ at repeated measurement according to 2007 American Heart Association Criteria (22); Diabetes is defined as fasting glucose $\geq 7 \mathrm{mmol} / \mathrm{L}$ or postprandial glucose $\geq 11.1 \mathrm{mmol} / \mathrm{L}$ and/or hemoglobin $\mathrm{A} 1 \mathrm{C} \geq 6.5 \%$ according to 2010 American Diabetes Association Criteria (23); Dyslipidemia is defined as having any one of the following four conditions: total cholesterol $(\mathrm{TC}) \geq 6.22 \mathrm{mmol} / \mathrm{L}$, triglycerides (TG) $\geq 2.26 \mathrm{mmol} / \mathrm{L}$, high-density lipoprotein cholesterol (HDLC) $<1.04 \mathrm{mmol} / \mathrm{L}$, low-density lipoprotein cholesterol (LDL-C) $\geq 4.14 \mathrm{mmol} / \mathrm{L}$ according to 2007 Chinese Guidelines on Prevention and Treatment of Dyslipidemia of Adults (24)), oral supplement use, exercise habit (more than three times/week and $60 \mathrm{~min} /$ time regular exercise), smoking and drinking alcohol habit were collected from each participant. BMI was calculated as the ratio of weight divided by height squared $\left(\mathrm{kg} / \mathrm{m}^{2}\right)$. Blood pressure (BP) was measured from the right arm using a standard $\mathrm{Hg}$ sphygmomanometer after $10 \mathrm{~min}$ rest with the patient in a sitting position. SBP was measured at Korotkoff phase I and DBP at Korotkoff phase V, following the recommendations of the American Heart Association. Patients' BP was measured three times, with an interval of more than $2 \mathrm{~min}$ between each measurement, and the mean value was used for analysis.

\section{Laboratory measurements}

Blood specimens were collected from all patients after overnight fasting of at least $8 \mathrm{~h}$. Fasting blood glucose

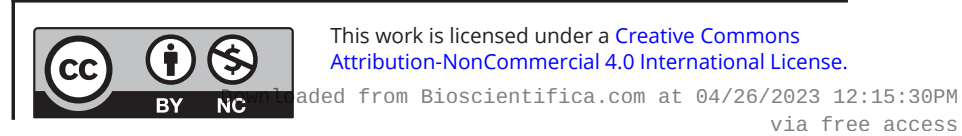


(FBG), insulin (IN), homeostasis model assessment-insulin resistance (HOMA-IR), aspartate aminotransferase (AST), alanine aminotransferase (ALT), TC, HDL-C, LDL-C, TG, creatinine $(\mathrm{Cr})$, uric acid (UA), free triiodothyronine (FT3), free thyroxine (FT4), thyroid-stimulating hormone (TSH), TT (total testosterone), and Hcy levels were recorded. Hcy values were determined with an assay kit (MAKER, China) based on the enzymatic cycling method, according to the manufacturer's instructions. The HOMA-IR was determined using the formula: HOMA-IR = $(\mathrm{FBG}(\mathrm{mmol} / \mathrm{L}) \times \mathrm{IN}(\mathrm{mU} / \mathrm{L})) / 22.5(25)$.

\section{BaPWV measurements}

The baPWV was recorded twice for each patient using a non-invasive vascular screening device (Omron Healthcare, Kyoto, Japan) by an experienced technician who was blinded to the cardiovascular risk profiles and Hcy levels of all the patients. The women rested in a supine position for $5 \mathrm{~min}$ before data collection. The baPWV value was calculated automatically according to the formula baPWV $=\mathrm{L} / \mathrm{PTT}$, in which $\mathrm{L}$ represented the difference in length from the heart to ankle (automatically calculated according to the formula $0.8129 \times$ height $(\mathrm{cm})+12.328)$ and the length from the heart to brachium (automatically calculated according to the formula $0.2195 \times$ height -2.0734$)$, and PTT was the pulse transit time between the brachial and tibial arterial waveforms (26). The mean values of right and left baPWV were recorded. Additionally, each woman was requested to measure the baPWV on another day within 2 months to assess the reproducibility of the index. One hundred and eight women completed the retest of baPWV. The reproducibility analyses include the means of individual between-visit coefficients of variation (CV) and intraclass correlation coefficients (ICC) using one-way ANOVA. The mean CV with 95\% CI was 3.71\% (3.38-4.25\%), and ICC with 95\% CI was 0.88 (0.73-0.95), which indicated that baPWV measurement was highly reproducible in those women.

\section{Statistics}

Descriptive statistics were presented as the mean \pm S.D. for continuous variables or percentage for categorical variables. The participants were divided into two groups according to baPWV values. Participants with a baPWV value $<1400 \mathrm{~cm} / \mathrm{s}$ were defined as normal, while those with a baPWV value $\geq 1400 \mathrm{~cm} / \mathrm{s}$ were defined as having high AS $(26,27,28)$. Comparisons between the two groups were performed using the Student's independent t-test (two-tailed) for normally distributed data or the Mann-Whitney $U$-test for parameters with a non-normal distribution. Count data (data expressed as the number of women) were analyzed using the $\chi^{2}$ test. A generalized linear model (univariate analysis) using the generalized estimating equation was performed separately to investigate the relative factors for baPWV. Then, multiple regression analysis was used to evaluate the association of Hcy with baPWV. The first model was adjusted for age, and the second model was adjusted for variables with a $P$-value $<0.05$ in the univariate analysis to assess whether circulating Hcy was independently associated with baPWV in women with PCOS. Differences with a two-sided $P$ value of $<0.05$ were considered statistically significant. All statistical analyses were performed using the SPSS software package (version 21.0; SPSS Inc.).

\section{Results}

Demographic, biochemical and clinical characteristics of 124 women with PCOS were presented in Table 1 . There was no woman having the habit of smoking or drinking alcohol. Thirty-five women (28.2\%) were divided into the group of high AS. Hypertension and diabetes were more frequent $(P<0.05)$ in the high AS group than the other one. There were significant differences in the ratio of taking omega fatty acid supplements, anti-hypertensive drugs, aspirin, sulfonylureas, and oral contraceptives between the two groups $(P<0.05)$. Age, BMI, PCOS duration, TG, LDL-C, and Hcy were higher in the group with high AS $(P<0.05)$, while there were no significant differences in the ratio of exercise, the ratio of taking metformin, the ratio of dyslipidemia, SBP, DBP, AST, ALT, FBG, IN, HOMA-IR, TC, HDL-C, Cr, UA, $\mathrm{FT}_{3}, \mathrm{FT}_{4}$, TSH, and TT between the two groups.

Univariate analysis (Table 2) revealed that age ( $r=0.254, P<0.01)$, BMI $(r=0.223, P<0.05)$, PCOS years $(r=0.104, P<0.05), \operatorname{SBP}(r=0.269, P<0.05), \mathrm{DBP}(r=0.162$, $P<0.05)$, HOMA-IR $(r=0.163, P<0.01)$, TC $(r=0.237$, $P<0.05)$, and Hcy $(r=0.133, P<0.05)$ were positively correlated with baPWV.

In multiple regression analysis (Table 3), the ageadjusted serum Hcy level was positively correlated with baPWV $(\beta=0.201, P<0.01)$. Serum Hcy remained positively associated with baPWV $(\beta=0.145, P<0.01)$ after further adjustments for BMI, PCOS duration, SBP, TC, and other factors that correlated with baPWV. 
Table 1 Demographic and clinical characteristics of the 124 PCOS women with normal baPWV or high baPWV.

\begin{tabular}{|c|c|c|c|}
\hline Parameters & $\begin{array}{c}\text { Women with } \\
\text { high baPWV } \\
\quad(n=35)\end{array}$ & $\begin{array}{c}\text { Women with } \\
\text { normal } \\
\text { baPWV } \\
(n=89)\end{array}$ & $P$-value \\
\hline Age (years) & $41.4 \pm 7.7$ & $35.8 \pm 7.9$ & 0.001 \\
\hline $\begin{array}{l}\text { PCOS duration } \\
\text { (months) }\end{array}$ & $116.4 \pm 18.1$ & $92.7 \pm 15.6$ & 0.008 \\
\hline $\mathrm{BMI}\left(\mathrm{kg} / \mathrm{m}^{2}\right)$ & $26.9 \pm 3.4$ & $27.1 \pm 2.6$ & 0.179 \\
\hline $\begin{array}{l}\text { Omega fatty acid } \\
\text { supplements }(n, \%)\end{array}$ & $7(20 \%)$ & $5(5.6 \%)$ & 0.002 \\
\hline Exercise habit $(n, \%)$ & $8(22.9 \%)$ & $18(20.2 \%)$ & 0.416 \\
\hline Hypertension $(n, \%)$ & $6(17.1 \%)$ & $7(7.9 \%)$ & 0.005 \\
\hline Diabetes $(n, \%)$ & $7(20 \%)$ & $7(7.9 \%)$ & 0.002 \\
\hline Dyslipidemia $(n, \%)$ & $28(80 \%)$ & $65(73.0 \%)$ & 0.486 \\
\hline $\begin{array}{l}\text { Anti-hypertensive } \\
\text { drugs }(n, \%)\end{array}$ & $5(14.3 \%)$ & $4(4.5 \%)$ & $<0.001$ \\
\hline Aspirin $(n, \%)$ & $6(17.1 \%)$ & $10(11.2 \%)$ & 0.017 \\
\hline Statins $(n, \%)$ & $13(37.1 \%)$ & $18(20.2 \%)$ & 0.002 \\
\hline Metformin $(n, \%)$ & $10(28.6 \%)$ & $20(22.5 \%)$ & 0.143 \\
\hline Sulfonylureas $(n, \%)$ & $4(11.4 \%)$ & $4(4.5 \%)$ & 0.007 \\
\hline $\begin{array}{l}\text { Oral contraceptives } \\
(n, \%)\end{array}$ & $5(14.3 \%)$ & $7(7.8 \%)$ & 0.004 \\
\hline SBP (mmHg) & $123.1 \pm 8.7$ & $124.6 \pm 7.1$ & 0.226 \\
\hline $\mathrm{DBP}(\mathrm{mmHg})$ & $78.7 \pm 6.2$ & $77.4 \pm 8.5$ & 0.604 \\
\hline $\operatorname{ALT}(\mathrm{U} / \mathrm{L})$ & $31.3 \pm 7.4$ & $32.9 \pm 6.3$ & 0.379 \\
\hline AST (U/L) & $27.2 \pm 6.8$ & $26.9 \pm 5.1$ & 0.427 \\
\hline FBG (mmol/L) & $5.1 \pm 0.7$ & $4.8 \pm 0.6$ & 0.264 \\
\hline IN (U/L) & $9.0(6.4-13.2)$ & $8.4(6.0-11.9)$ & 0.321 \\
\hline HOMA-IR & $1.6(1.3-2.4)$ & $1.7(1.4-2.6)$ & 0.593 \\
\hline $\mathrm{TC}(\mathrm{mmol} / \mathrm{L})$ & $5.7 \pm 1.1$ & $5.6 \pm 1.5$ & 0.205 \\
\hline TG (mmol/L) & $2.2 \pm 0.8$ & $2.0 \pm 0.9$ & 0.006 \\
\hline HDL-C (mmol/L) & $1.3 \pm 0.4$ & $1.2 \pm 0.5$ & 0.744 \\
\hline LDL-C (mmol/L) & $3.7 \pm 1.1$ & $3.0 \pm 1.0$ & 0.018 \\
\hline $\mathrm{Cr}(\mu \mathrm{mol} / \mathrm{L})$ & $72.1 \pm 11.8$ & $75.8 \pm 10.0$ & 0.253 \\
\hline $\mathrm{UA}(\mu \mathrm{mol} / \mathrm{L})$ & $328.4 \pm 21.9$ & $317.2 \pm 24.8$ & 0.372 \\
\hline $\mathrm{FT}_{3}(\mathrm{pmol} / \mathrm{L})$ & $4.1 \pm 1.1$ & $4.3 \pm 1.4$ & 0.504 \\
\hline $\mathrm{FT}_{4}(\mathrm{pmol} / \mathrm{L})$ & $16.8 \pm 2.7$ & $17.2 \pm 3.1$ & 0.366 \\
\hline $\mathrm{TSH}(\mathrm{mlU} / \mathrm{L})$ & $2.5 \pm 0.8$ & $2.2 \pm 1.0$ & 0.115 \\
\hline TT (nmol/L) & $2.6(1.8-3.6)$ & $2.8(1.9-3.9)$ & 0.281 \\
\hline Hcy ( $\mu \mathrm{mol} / \mathrm{L})$ & $12.3(8.5-16.8)$ & $9.0(6.5-13.1)$ & 0.027 \\
\hline
\end{tabular}

ALT, alanine aminotransferase; AST, aspartate aminotransferase; baPWV, brachial-ankle pulse wave velocity; $\mathrm{Cr}$, creatine; DBP, diastolic blood pressure; FBG, fasting blood glucose; FT3, free triiodothyronine; FT4, free thyroxine; Hcy, homocysteine; Hcy, homocysteine; HDL-C, high-density lipoprotein cholesterol; HOMA-IR, homeostasis model assessment-insulin resistance; IN, insulin; LDL-C, low-density lipoprotein cholesterol; SBP, systolic bloodpressure; TC, total cholesterol; TG, triglyceride; TSH, thyroid-stimulating hormone; TT, total testosterone; UA, uric acid.

\section{Discussion}

To our knowledge, this cross-sectional study is the first to reveal that Hcy is an independent indicator of baPWV in Chinese women with PCOS. A growing number of investigations have confirmed that PCOS women exhibit an elevated prevalence of clinical and subclinical $\mathrm{CVD}$, such as increased carotid intima-media thickness,
Table 2 Univariate analysis of risk factors for baPWV based on demographic characteristics and biochemical parameters.

\begin{tabular}{l} 
Parameters \\
\hline Clinical variables \\
\hline Age (years) \\
BMI (kg/m²) \\
PCOS duration \\
(months) \\
SBP (mmHg) \\
DBP (mmHg) \\
FBG (mmol/L) \\
HOMA-IR \\
TC (mmol/L) \\
LDL-C (mmol/L) \\
UA (umol/L) \\
Hcy (umol/L) \\
\hline Demographic \\
characteristics \\
\hline Exercise habit \\
Hypertension \\
Diabetes \\
Dyslipidemia
\end{tabular}

\begin{tabular}{|c|c|}
\hline \multicolumn{2}{|c|}{ baPWV $(\mathrm{cm} / \mathrm{s})$} \\
\hline $\begin{array}{c}\text { Regression coefficient } \\
\text { with } 95 \% \mathrm{Cl}\end{array}$ & $P$-value \\
\hline $0.254(0.211-0.286)$ & 0.002 \\
\hline $0.223(0.206-0.251)$ & 0.036 \\
\hline $0.104(0.075-0.132)$ & 0.029 \\
\hline $0.269(0.214-0.293)$ & 0.018 \\
\hline $0.162(0.138-0.194)$ & 0.023 \\
\hline $0.138(0.095-0.180)$ & 0.605 \\
\hline $0.163(0.142-0.195)$ & 0.003 \\
\hline $0.237(0.206-0.287)$ & 0.014 \\
\hline $0.161(0.120-0.213)$ & 0.582 \\
\hline $0.296(0.242-0.371)$ & 0.317 \\
\hline $0.133(0.108-0.168)$ & 0.011 \\
\hline F value with $95 \% \mathrm{Cl}$ & P-value \\
\hline $0.912(0.803-1.147)$ & 0.267 \\
\hline $2.321(2.105-2.486)$ & 0.006 \\
\hline $3.014(2.863-3.352)$ & 0.012 \\
\hline 1.205 (1.017-1.529) & 0.228 \\
\hline
\end{tabular}

endothelial dysfunction, and AS (3). According to the recently published international PCOS guidelines, global CVD risk should be routinely assessed in women with PCOS (3). Besides fasting glucose or glycosylated hemoglobin, lipid profiles and BP should be monitored annually, some additional tests are advised to serve as tiebreakers for further stratifying those women (13). The question, which is whether the broader application of investigating Hcy levels and treating H-Hcy in PCOS women would favor the course of PCOS management in the long run, was raised

Table 3 Multiple linear analysis with baPWV (dependent variables) and Hcy (independent variables).

\begin{tabular}{|c|c|c|}
\hline Parameter & $\beta$ value with $95 \% \mathrm{Cl}$ & P-value \\
\hline \multicolumn{3}{|l|}{ Model $1^{a}$} \\
\hline Age & $0.312(0.207-0.426)$ & 0.003 \\
\hline Hcy & $0.201(0.129-0.385)$ & 0.002 \\
\hline \multicolumn{3}{|l|}{ Model $2^{b}$} \\
\hline Age & $0.217(0.116-0.409)$ & 0.025 \\
\hline $\mathrm{Hcy}$ & $0.145(0.083-0.182)$ & 0.007 \\
\hline BMI & $0.268(0.142-0.371)$ & 0.379 \\
\hline SBP & $0.291(0.198-0.353)$ & 0.002 \\
\hline HOMA-IR & $0.429(0.304-0.576)$ & 0.273 \\
\hline $\mathrm{TC}$ & $0.102(0.051-0.227)$ & 0.536 \\
\hline Hypertension & $0.163(0.080-0.245)$ & 0.024 \\
\hline Diabetes & $0.248(0.113-0.368)$ & 0.261 \\
\hline
\end{tabular}

${ }^{a}$ Model 1 adjusted for age, Hcy; ${ }^{b}$ Model 2 adjusted for parameters that had a $P$ value $<0.05$ in the univariate analysis of risk factors for baPWV. $\beta$, standardized regression coefficient; Hcy, homocysteine; HOMA-IR, homeostasis model assessment-insulin resistance; SBP, systolic blood pressure; TC, total cholesterol.

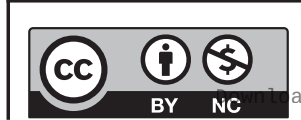


by clinicians. Our efforts were to collect the data of Hcy in Chinese PCOS women. Longitudinal studies with a larger sample size are needed to determine whether it's helpful to include testing Hcy level in routine clinical practice.

AS results from a degenerative process that mainly affects the extracellular matrix of elastic arteries due to the effects of aging and other risk factors (27). It has been widely studied in different clinical settings, and AS is emerging as an interesting tissue biomarker for CVD risk stratification and estimation of an individual's 'biological age' (27). As women with PCOS are at greater risk of developing CVD, research has been carried out to study the prevalence of AS in women of different ages. Armeni et al. reported that AS, evaluated by pulse wave velocity and augmentation index, was increased in asymptomatic nondiabetic postmenopausal women (mean age 55.6 \pm 7.8 years) with PCOS (7). Similarly, AS measured by the cardioankle vascular index was found to be greater in young nonobese women with PCOS (mean age $22.90 \pm 3.04$ years) compared to subjects without PCOS (6). Besides age, BP has been verified as another common factor that influences AS (27). A previous investigation based in Japan revealed that AS evaluated by baPWV in mildly hypertensive women (SBP $\geq 120 \mathrm{mmHg}$ or $\mathrm{DBP} \geq 90 \mathrm{mmHg}$ ) with PCOS (mean age $32.6 \pm 2.6$ years) was significantly higher than that in healthy controls (mean age $22.90 \pm 3.04$ years) or normotensive women with PCOS (mean age $31.5 \pm 4.4$ years) (9). Our finding confirmed that age and HBP, especially SBP level, were closely related to AS in PCOS women.

The measurement of baPWV, which is easy and powerful, has been widely used in East Asian countries over the past 2 decades (28). A meta-analysis of cohort studies conducted in the general population, with hypertension, diabetes, end-stage renal disease, and other high-risk individuals, showed that a $1 \mathrm{~m} / \mathrm{s}$ increase in baPWV is associated with a $12 \%$ increase in the risk of CVD events (27). Regarding the importance of baPWV in the assessment of AS, it was also used to evaluate the effects of treatment in these women. Takahashi et al. found that baPWV was an independent prognostic factor for the ovulatory response to clomiphene citrate, which is the first-line strategy to induce ovulation in women with PCOS (8). But only a few analogic studies focused on baPWV values in women with PCOS in our country, where the prevalence of PCOS in women aged 19-45 years has been reported to be about $5.6 \%$ (29). This large community-based study included a total of 16,886 women from 152 cities and 112 villages (29). However, more and more epidemiological studies have investigated the role of AS (adopting baPWV $\geq 1400 \mathrm{~cm} / \mathrm{s}$ as the cutoff) in different Chinese female populations, such as apparently healthy middle-aged women (30), women with a history of preeclampsia (19), and women with diabetes (31). The present study was the first to collect the arterial compliance information of women with PCOS in China, which might be helpful to provide more comprehensive risk stratification management of CVD in this special population.

The nature of the possible link between AS and atherosclerosis has not been clearly established due to the fact that their inter-relationship is complex and may involve a number of different hemodynamic, mechanical, metabolic, and enzymatic mechanisms (27). Soares let al. found that young women with PCOS may present with vascular elasticity changes even in the absence of classical risk factors for CVD such as hypertension and obesity (32). Hcy, a sulfur-containing amino acid formed during the metabolism of methionine, exerts cytotoxic effects on the vascular endothelium (13). McCully (33) was the first to propose that Hcy played a role in the pathogenesis of arteriosclerosis, which was subsequently verified by numerous studies. Three decades later, the 'Hcy-CVD' hypothesis was proposed by Clarke and colleagues (34), with an odds ratio of 3.2 for CVD. Accumulating literature has documented positive relationships between H-Hcy and AS in diabetic patients (15), hypertensive patients (18), and in community-based general populations (17). Nevertheless, there are limited data on the link between H-Hcy and AS in PCOS women. We found that serum Hcy was positively and independently associated with baPWV, although the underlying mechanism was outside the scope of our study. Preliminary investigations in women with PCOS demonstrated correlations between H-Hcy and various components of PCOS, such as androgen excess and reduced ovulation, as well as cardiovascular health (13). These positive findings led clinicians to further explore associations between H-Hcy and insulin resistance (35), oxidative stress (36), and chronic inflammation (37). Whether Hcy is a potential therapeutic target to improve AS in PCOS needs experimental designs in animals or related interventions in humans to provide such mechanistic insight.

Despite the interesting findings obtained by the current study, certain limitations should be considered. First, this was a single-center study in which only a small number of women with PCOS were available for analysis. Secondly, the present study was cross-sectional. Serial Hcy and baPWV values would be more meaningful to explore the cause and effect. Thirdly, we did not collect all the medicine information they were taking, which might

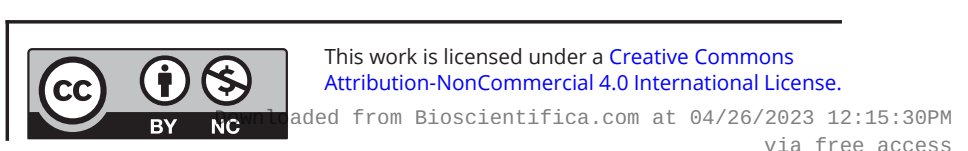


have a potential influence on baPWV values. Those factors would also be recorded and justified in further studies.

\section{Conclusions}

In conclusion, our study demonstrated that serum Hcy levels were positively and independently associated with baPWV in Chinese women with PCOS. Further investigations with a larger sample size are needed to explore the possibility of including Hcy level as a biomarker for the serial stratified prevention and management of CVD in those women.

\section{Declaration of interest}

The authors declare that there is no conflict of interest that could be perceived as prejudicing the impartiality of the research reported.

\section{Funding}

This work was partly supported by Youth Project on Health Scientific Research in Jing'an District, Shanghai (2017QN02).

\section{Acknowledgements}

The authors thank all the patients and parents for their kind participation in our study.

\section{References}

1 Lizneva D, Suturina L, Walker W, Brakta S, Gavrilova-Jordan L \& Azziz R. Criteria, prevalence, and phenotypes of polycystic ovary syndrome. Fertility and Sterility 2016106 6-15. (https://doi. org/10.1016/j.fertnstert.2016.05.003)

2 Orio F, Muscogiuri G, Nese C, Palomba S, Savastano S, Tafuri D, Colarieti G, La Sala G, Colao A \& Yildiz BO. Obesity, type 2 diabetes mellitus and cardiovascular disease risk: an up to date in the management of polycystic ovary syndrome. European Journal of Obstetrics, Gynecology, and Reproductive Biology 2016207 214-219. (https://doi.org/10.1016/j.ejogrb.2016.08.026)

3 Osibogun O, Ogunmoroti O \& Michos ED. Polycystic ovary syndrome and cardiometabolic risk: opportunities for cardiovascular disease prevention. Trends in Cardiovascular Medicine 202030 399-404. (https://doi.org/10.1016/j.tcm.2019.08.010)

4 Ohkuma T, Ninomiya T, Tomiyama H, Kario K, Hoshide S, Kita Y, Inoguchi T, Maeda Y, Kohara K, Tabara Y, et al. Brachial-ankle pulse wave velocity and the risk prediction of cardiovascular disease: an individual participant data meta-analysis. Hypertension 201769 1045-1052. (https://doi.org/10.1161/HYPERTENSIONAHA.117.09097)

5 Kim J, Choi SY, Park B, Park HE, Lee H, Kim MJ, Kim SM, Hwang KR \& Choi YM. Arterial stiffness measured by cardio-ankle vascular index in Korean women with polycystic ovary syndrome. Journal of Obstetrics and Gynaecology 201939 681-686. (https://doi.org/10.1080/01443615. 2018.1561654)

6 Kilic D, Kilic ID, Sevgican CI, Kilic O, Alatas E, Arslan M, Avci E \& Guler T. Arterial stiffness measured by cardio-ankle vascular index is greater in non-obese young women with polycystic ovarian syndrome.
Journal of Obstetrics and Gynaecology Research 202147 521-528. (https://doi.org/10.1111/jog.14543)

7 Armeni E, Stamatelopoulos K, Rizos D, Georgiopoulos G, Kazani M, Kazani A, Kolyviras A, Stellos K, Panoulis K, Alexandrou A, et al. Arterial stiffness is increased in asymptomatic nondiabetic postmenopausal women with a polycystic ovary syndrome phenotype. Journal of Hypertension 201331 1998-2004. (https://doi. org/10.1097/HJH.0b013e3283630362)

8 Takahashi T, Igarashi H, Hara S, Amita M, Matsuo K, Hasegawa A \& Kurachi H. Brachial-to-ankle pulse wave velocity as an independent prognostic factor for ovulatory response to clomiphene citrate in women with polycystic ovary syndrome. Journal of Ovarian Research 20147 74. (https://doi.org/10.1186/1757-2215-7-74)

9 Sasaki A, Emi Y, Matsuda M, Sharula, Kamada Y, Chekir C, Hiramatsu Y \& Nakatsuka M. Increased arterial stiffness in mildly-hypertensive women with polycystic ovary syndrome. Journal of Obstetrics and Gynaecology Research 201137 402-411. (https://doi.org/10.1111/j.14470756.2010.01375.x)

10 de Koning EJ, van der Zwaluw NL, van Wijngaarden JP, Sohl E, Brouwer-Brolsma EM, van Marwijk HW, Enneman AW, Swart KM, van Dijk SC, Ham AC, et al. Effects of two-year vitamin B12 and folic acid supplementation on depressive symptoms and quality of life in older adults with elevated homocysteine concentrations: additional results from the b-proof study, an RCT. Nutrients 20168 E748. (https://doi. org/10.3390/nu8110748)

11 Cellai AP, Lami D, Antonucci E, Liotta AA, Rogolino A, Fedi S, Fiorillo C, Becatti M, Cenci C, Marcucci R, et al. Hyperhomocysteinemia in patients with pulmonary embolism is associated with impaired plasma fibrinolytic capacity. Journal of Thrombosis and Thrombolysis 201438 45-49. (https://doi.org/10.1007/s11239-013-0981-1)

12 Huo Y, Li J, Qin X, Huang Y, Wang X, Gottesman RF, Tang G, Wang B, Chen D, He M, et al. Efficacy of folic acid therapy in primary prevention of stroke among adults with hypertension in China: the CSPPT randomized clinical trial. JAMA 2015313 1325-1335. (https:// doi.org/10.1001/jama.2015.2274)

13 Kondapaneni V, Gutlapalli SD, Poudel S, Zeb M, Toulassi IA \& Cancarevic I. Significance of homocysteine levels in the management of polycystic ovarian syndrome: a literature review. Cureus 202012 e11110. (https://doi.org/10.7759/cureus.11110)

14 Tayama J, Munakata M, Yoshinaga K \& Toyota T. Higher plasma homocysteine concentration is associated with more advanced systemic arterial stiffness and greater blood pressure response to stress in hypertensive patients. Hypertension Research 200629 403-409. (https://doi.org/10.1291/hypres.29.403)

15 Shargorodsky M, Boaz M, Pasternak S, Hanah R, Matas Z, Fux A Beigel Y \& Mashavi M. Serum homocysteine, folate, vitamin B12 levels and arterial stiffness in diabetic patients: which of them is really important in atherogenesis? Diabetes/Metabolism Research and Reviews 200925 70-75. (https://doi.org/10.1002/dmrr.902)

16 Ruan L, Chen W, Srinivasan SR, Xu J, Sun M, Toprak A \& Berenson GS Relation of plasma homocysteine to arterial stiffness in black and white young adults (from the Bogalusa Heart Study). American Journal of Cardiology 2009103 985-988. (https://doi.org/10.1016/j. amjcard.2008.12.012)

17 Zhang S, Bai YY, Luo LM, Xiao WK, Wu HM \& Ye P. Association between serum homocysteine and arterial stiffness in elderly: a community-based study. Journal of Geriatric Cardiology 201411 32-38. (https://doi.org/10.3969/j.issn.1671-5411.2014.01.007)

18 Liu J, Liu H, Zhao H, Zhou Y, Li L \& Wang H. Relationship between cardio-ankle vascular index and homocysteine in hypertension subjects withhyperhomocysteinemia. Clinical and Experimental Hypertension 201638 652-657. (https://doi.org/10.1080/10641963.2016 .1182183)

19 Wu F, Yang H \& Liu B. Association between homocysteine and arterial stiffness in women with a history of preeclampsia. Journal of Vascular Research 201956 152-159. (https://doi.org/10.1159/000500358) 
20 Rotterdam ESHRE/ASRM-Sponsored PCOS Consensus Workshop Group. Revised 2003 consensus on diagnostic criteria and long-term health risks related to polycystic ovary syndrome (PCOS). Human Reproduction 200419 41-47. (https://doi.org/10.1093/humrep/deh098)

21 Hulley SB. Designing Clinical Research. Lippincott Williams \& Wilkins, 2013.

22 Kotchen TA. Developing hypertension guidelines: an evolving process. American Journal of Hypertension 201427 765-772. (https://doi. org/10.1093/ajh/hpt298)

23 Kumar R, Nandhini LP, Kamalanathan S, Sahoo J \& Vivekanadan M. Evidence for current diagnostic criteria of diabetes mellitus. World Journal of Diabetes 20167 396-405. (https://doi.org/10.4239/wjd. v7.i17.396)

24 Joint Committee for Developing Chinese guidelines on Prevention and Treatment of Dyslipidemia in Adults. Chinese guidelines on prevention and treatment of dyslipidemia in adults. Zhonghua Xin Xue Guan Bing Za Zhi 200735 390-419.

25 Diamanti-Kandarakis E, Spritzer PM, Sir-Petermann T \& Motta AB. Insulin resistance and polycystic ovary syndrome through life. Current Pharmaceutical Design 201218 5569-5576. (https://doi. org/10.2174/138161212803307590)

26 Yan N, Zhou Y, Wang Y, Wang A, Yang X, Russell A, Wu S, Zhao X \& Wang W. Association of ideal cardiovascular health and brachial-ankle pulse wave velocity: a cross-sectional study in northern China. Journal of Stroke and Cerebrovascular Diseases 201625 41-48. (https://doi. org/10.1016/j.jstrokecerebrovasdis.2015.08.031)

27 Palombo C \& Kozakova M. Arterial stiffness, atherosclerosis and cardiovascular risk: pathophysiologic mechanisms and emerging clinical indications. Vascular Pharmacology 201677 1-7. (https://doi. org/10.1016/j.vph.2015.11.083)

28 Munakata M. Brachial-ankle pulse wave velocity in the measurement of arterial stiffness: recent evidence and clinical applications. Current Hypertension Reviews 201410 49-57. (https://doi.org/10.2174/15734021 1001141111160957)

29 Li R, Zhang Q, Yang D, Li S, Lu S, Wu X, Wei Z, Song X, Wang X, Fu S, et al. Prevalence of polycystic ovary syndrome in women in China: a large community-based study. Human Reproduction 201328 2562-2569. (https://doi.org/10.1093/humrep/det262)

30 Wen JH, Zhong YY, Wen ZG, Kuang CQ, Liao JR, Chen LH, Wang PS, Wu YX, Ouyang CJ \& Chen ZJ. Triglyceride to HDL-C ratio and increased arterial stiffness in apparently healthy individuals. International Journal of Clinical and Experimental Medicine 20158 4342-4348.

31 Tan JR, Chen YH, Bi YF, Xu M, Huang Y, Dai M, Ning G \& Li XY. Prehypertension is associated with atherosclerosis in type 2 diabetes. Journal of Diabetes 20102 56-63. (https://doi.org/10.1111/j.17530407.2009.00062.x)

32 Soares GM, Vieira CS, Martins WP, Franceschini SA, dos Reis RM, Silva de Sá MF \& Ferriani RA. Increased arterial stiffness in nonobese women with polycystic ovary syndrome (PCOS) without comorbidities: one more characteristic inherent to the syndrome? Clinical Endocrinology 200971 406-411. (https://doi.org/10.1111/j.1365-2265.2008.03506.x)

33 McCully KS. Vascular pathology of homocysteinemia: implications for the pathogenesis of arteriosclerosis. American Journal of Pathology 1969 56 111-128.

34 Clarke R, Daly L, Robinson K, Naughten E, Cahalane S, Fowler B \& Graham I. Hyperhomocysteinemia: an independent risk factor for vascular disease. New England Journal of Medicine 1991324 1149-1155. (https://doi.org/10.1056/NEJM199104253241701)

35 Chakraborty P, Goswami SK, Rajani S, Sharma S, Kabir SN, Chakravarty B \& Jana K. Recurrent pregnancy loss in polycystic ovary syndrome: role of hyperhomocysteinemia and insulin resistance. PLoS ONE 20138 e64446. (https://doi.org/10.1371/journal.pone.0064446)

36 Murri M, Luque-Ramírez M, Insenser M, Ojeda-Ojeda M \& EscobarMorreale HF. Circulating markers of oxidative stress and polycystic ovary syndrome (PCOS): a systematic review and meta-analysis. Human Reproduction Update 201319 268-288. (https://doi.org/10.1093/ humupd/dms059)

37 Guzelmeric K, Alkan N, Pirimoglu M, Unal O \& Turan C. Chronic inflammation and elevated homocysteine levels are associated with increased body mass index in women with polycystic ovary syndrome. Gynecological Endocrinology 200723 505-510. (https://doi. org/10.1080/09513590701554306)

Received in final form 20 July 2021

Accepted 6 August 2021

Accepted Manuscript published online 6 August 2021
This work is licensed under a Creative Commons Attribution-NonCommercial 4.0 International License. ded from Bioscientifica.com at 04/26/2023 12:15:30pM 